\title{
Significance of Ca-soap formation for calcium absorption in the rat
}

\author{
G. GACS AND D. BARLTROP 1 \\ From the Paediatric Unit, St. Mary's Hospital Medical School, London
}

SUMMARY The significance of calcium soap formation in the inhibition of calcium absorption has been studied in rats. ${ }^{47} \mathrm{Ca}$ labelled soaps of fatty acids were introduced into the duodenum and the absorption of calcium measured after four hours in a whole body counter. The absorption of calcium was inversely correlated with the chain length of the fatty acid varying from $1 \%$ for Ca-stearate to $60 \%$ for Ca-hexanoate. Increasing the degree of unsaturation of the fatty acid was accompanied by increased calcium absorption. The availability of calcium for absorption from the soaps was correlated with their solubility in $1 \%$ aqueous Na-tauroglycocholate. The percentages of calcium as soap in the small intestine and the faeces after intragastric administration of calcium and fats were similar, which suggests that the faecal content of calcium soaps is an index of intestinal soap formation. Soap formation was negligible when $\mathrm{CaCl}_{2}$ was given with tristearate, trioleate, or tridecanoate and no depression of calcium absorption was observed. Calcium absorption was markedly impaired by the addition of phosphates at a $\mathrm{Ca} / \mathrm{P}$ ratio of $1: 1$ irrespective of the presence of neutral fats. Stearic acid resulted in significant soap formation and reduced calcium absorption. The degree of Ca-soap formation and the inhibition of calcium absorption were well correlated. The results suggest that, although calcium soap formation may markedly depress calcium absorption in the rat, no significant soap formation takes place when fats are given in the form of triglycerides.

The influence of dietary fat on calcium absorption has been extensively studied since the beginning of the century and there is an extensive literature (Boyd et al., 1932; Tadayyon and Lutwak, 1969). It has been consistently observed that increased faecal fat excretion is accompanied by increased calcium loss both in man (Givens, 1917; Widdowson, 1965; Kehayoglou et al., 1968) and in rat (Tadayyon and Lutwak, 1969; Kehayoglou et al., 1968).

It remains uncertain, however, whether a causal relationship between fat absorption and calcium loss exists. It has been suggested that long chain saturated fatty acids form insoluble soaps with calcium in the intestine thus preventing its absorption (Widdowson, 1965). The extent of absorption of calcium from Ca-stearate, Ca-oleate, and $\mathrm{Ca}$ palmitate has been studied by Boyd et al. (1932) and found to be related to the nature of the fatty icid

${ }^{1}$ Address for correspondence: Dr D. Barltrop, Paediatric Unit, St. Mary's Hospital Medical School, London, W2 1PG.

Received for publication 19 July 1976 components. Nicolaysen (1943) reinvestigated this problem and concluded that the calcium absorption from the corresponding soaps was largely independent of the nature of the fatty acid. These studies, however, did not take into account the splitting of Ca-soaps in the diet that may have occurred in the acid milieu of the stomach so that some of the calcium may have entered the duodenum in the ionised form.

In this study the availability of calcium for absorption from the soaps of a wide range of fatty acids has been measured. To avoid acid hydrolysis in the stomach, the soaps were introduced directly into the duodenum. In an attempt to explain the marked differences that were found, the solubility of Ca-soaps in a solution of bile salts was also studied. To investigate the possibility of soap formation from normal dietary constituents calcium absorption was determined after the intragastric administration of calcium chloride, together with three neutral fats in the presence and absence of dietary phosphate. The proportion of calcium as calcium soap was measured in intestinal contents and faeces using a similar technique. 


\section{Methods}

The fatty acid-calcium soaps were synthesised from $\mathrm{CaCl}_{2}$ labelled with ${ }^{47} \mathrm{Ca}$ (Amersham) and fatty acids in two steps through the sodium soaps. The soaps were washed with water and alcohol to remove unreacted calcium and fatty acids. Before use the soaps were emulsified in water by means of ultrasound. Ca-oleate and linoleate could only be emulsified in $0.05 \mathrm{M}$ Na-lauryl-sulphate, which in this concentration was found to have no effect on the absorption of calcium from other soaps. Soaps were prepared of saturated fatty acids of chain length $\mathrm{C} 6: 0-\mathrm{C} 18: 0$ and of two unsaturated fatty acids $C 18: 1$ and $C 18: 2$.

Male rats of $50-70 \mathrm{~g}$ body weight were used. Each soap was administered to groups of five to 10 animals. The experiments were performed under pentobarbitone (Nembutal) anaesthesia after overnight fasting. The duodenum was exposed and ligated immediately distal to the pylorus but the bile duct was preserved intact. The soaps were introduced into the intestine distal to the ligature. Each animal was given an amount of soap containing $4 \mathrm{mg}$ calcium. After four hours the animals were killed and the gastrointestinal tract removed. Calcium absorption was determined by the measurement of radioactivity in the carcass in a PackardArmac whole body counter for small animals.

For the determination of solubility, $100 \mathrm{mg}$ of each soap was suspended in $10 \mathrm{ml} 0.02 \mathrm{M} \mathrm{Na}$ tauroglycocholate, the $\mathrm{pH}$ adjusted to 6.5 with $\mathrm{NaOH}$ and the suspension shaken for two hours at $37^{\circ} \mathrm{C}$. Thereafter it was filtered (Whatman No. 40) and the calcium concentration measured in the filtrate by atomic absorption spectrophotometry.

For the study of the effect of fat on calcium absorption and intestinal soap formation, rats were given only water for 48 hours, after which $1.0 \mathrm{ml}$ of the calcium-fat mixture was introduced into the stomach under light ether anaesthesia. The emulsion administered contained $2.8 \mathrm{mg} \mathrm{CaCl}_{2}(1 \mathrm{mg} \mathrm{Ca})$ labelled with ${ }^{47} \mathrm{Ca}, 200 \mathrm{mg}$ fat, and $10 \mu \mathrm{l}$ Tween-80. The fats studied were the triglycerides of stearic, oleic, and decanoic acids. For comparison, similar studies were made in which stearic acid was substituted for the neutral fat. Control animals received $1 \mathrm{mg}$ calcium as the chloride alone. The potential interaction of dietary phosphates was explored by repeating these studies using calcium chloride to which sodium dihydrogen phosphate had been added to achieve a $\mathrm{Ca} / \mathrm{P}$ ratio of $1: 1$.

Half of the animals were killed after 90 minutes, the gut removed, and the contents of the small intestine washed out with distilled water. The ratio of soap to non-soap calcium was determined in the material collected from the intestine. The remaining animals were housed individually in metabolic cages and urine and faeces collected for 28 hours. Calcium absorption was calculated from the radioactivity measured in the carcass, gut, and faeces. The excretion of labelled calcium in the urine was negligible.

The determination of calcium soaps in the intestinal contents and in the faeces was based on the method of Sammons and Wiggs (1960) who used diethylether for their separation. By means of labelled calcium salts and soaps it was found however, that a substantial proportion of the calcium salts were trapped at the water-ether interphase and could not be separated from the calcium soaps at the bottom of the ether phase. Part of the soap was also found in the water phase. The method was therefore modified by the addition of sucrose to the homogenate to a final concentration of $0.06 \mathrm{M}$ before extraction with diethylether in a separation funnel. The ether phase was washed three times with water and the radioactivity measured in both fractions. In some of the samples the soaps were isolated, washed several times with ether and, after acid hydrolysis, the stable calcium and fatty acid contents determined. A mean fatty acid:calcium ratio of 15.6:1 was found which was close to the expected ratio of $14: 1$. The recovery of labelled Ca-stearate added to faeces was $85-90 \%$ and that of Ca-decanoate $75-80 \%, 3-8 \%$ of labelled calcium was recovered from the soap fraction.

\section{Results}

The absorption of calcium from $\mathrm{CaCl}_{2}$ was similar when introduced into the duodenum, the stomach, or mixed into the diet, showing that the experimental conditions did not modify the rate of absorption of ionised calcium.

The absorption of calcium from soaps was inversely related to chain length of the fatty acid component (Fig. 1). Thus, negligible absorption was observed from the soaps of long chain length saturated fatty acids but a progressive increase in absorption occurred with the decreasing of chain length. Increasing the degree of unsaturation of the component fatty acid was associated with enhanced calcium absorption (Fig. 2).

The solubility of calcium soaps in vitro resembled the absorption of calcium observed in vivo and was inversely correlated with the chain length and the degree of saturation of the constituent fatty acids (Fig. 3).

When calcium was given as $\mathrm{CaCl}_{2}$ and fat as triglyceride, calcium absorption was not depressed by any of the triglycerides compared with $\mathrm{CaCl}_{2}$ 


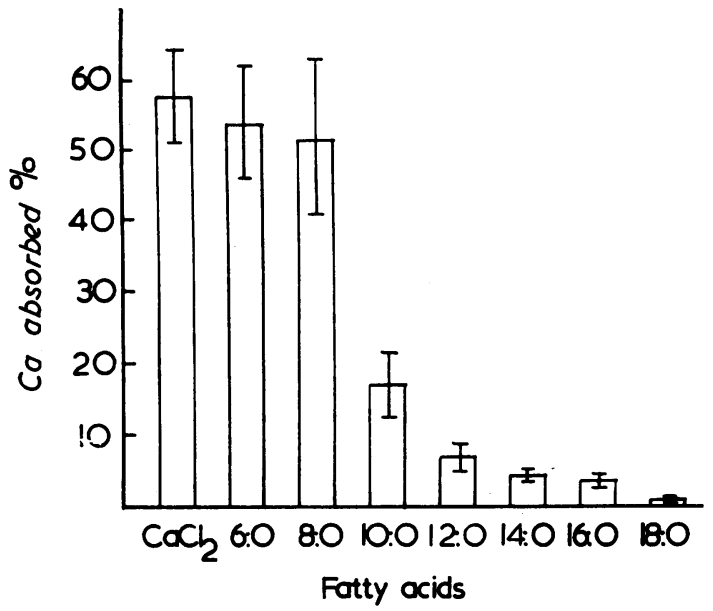

Fig. 1 Calcium absorption from the Ca-soaps of saturated fatty acids. Mean $\pm S D$.

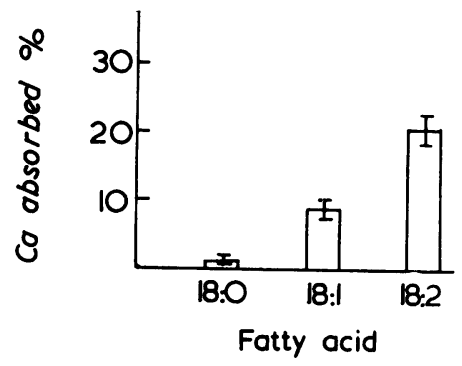

Fig. 2 Effect of unsaturation of fatty acids on the calcium absorption from their Ca-soaps. Mean $\pm S D$.

(Table 1). In the presence of phosphate $(\mathrm{Ca}: \mathrm{P}=1: 1)$ calcium absorption was markedly reduced, but again the triglycerides had no effect. By contrast, the administration of stearic acid instead of tri-

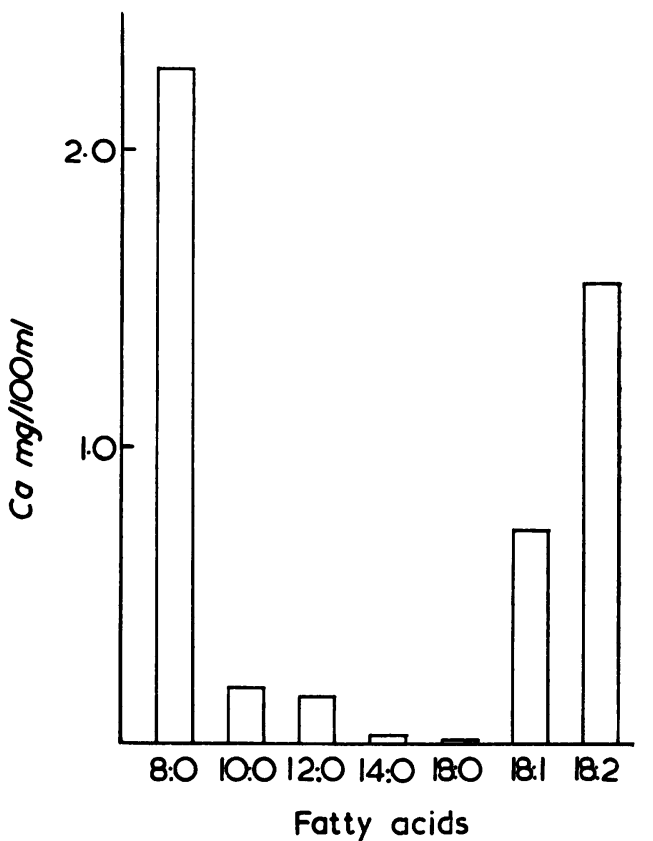

Fig. 3 Solubility of Ca-soaps in 0.02M Na-tauroglycocholate.

stearate resulted in a significant depression of calcium absorption.

The percentages of calcium as soap were very similar in the intestine and in the faeces with each fat studied. Soap formation was negligible with the triglycerides but was marked with stearic acid (Table 2). The combined data for tristearate, trioleate, and stearic acid showed a significant inverse correlation between the absorption of calcium and the amount of calcium soap in the faeces (Fig. 4). In the case of tristearate, $22.1 \%$ of the fat in the faeces was in the form of fatty acid or soap and $77.9 \%$ as neutral fat.

Table 1 Calcium absorbed \% ingested dose after intragastric administration of $1 \mathrm{mg}$ Ca and $100 \mathrm{mg}$ fat at different $\mathrm{Ca} / \mathrm{P}$ ratios

\begin{tabular}{llllll}
\hline CalP & - & Tristearate & Trioleate & Tridecanoate & Stearic acid \\
\hline $1: 0$ & $66.6 \pm 8 \cdot 6(8)$ & $59 \cdot 8 \pm 14 \cdot 2(15)$ & $59 \cdot 3 \pm 10 \cdot 3(8)$ & $\overline{32.0} \pm 7.3(6)$ & $32.0 \pm 7 \cdot 7(11)$ \\
$1: 1$ & $33.4 \pm 9.9(6)$ & $29.8 \pm 6.5(6)$ & $40.4 \pm 7.3(6)$ & - & \\
\hline
\end{tabular}

Mean $\pm \mathrm{SD}$. Number of animals in parentheses.

Table 2 Calcium \% as calcium soap in faeces and small intestine after intragastric administration of $1 \mathrm{mg}$ Ca and $200 \mathrm{mg}$ fat

\begin{tabular}{|c|c|c|c|c|c|}
\hline & - & Tristearate & Trioleate & Tridecanoate & Stearic acid \\
\hline $\begin{array}{l}\text { Faeces } \\
\text { Intestine }\end{array}$ & $\begin{array}{l}0-1(6) \\
0-1(6)\end{array}$ & $\begin{array}{l}6.5 \pm 3.8(14) \\
6.7 \pm 6.6(11)\end{array}$ & $\begin{array}{l}8 \cdot 0 \pm 4 \cdot 7(8) \\
6.7 \pm 1 \cdot 1(9)\end{array}$ & $\overline{6.4} \pm 1.8(12)$ & $\begin{array}{l}47 \cdot 7 \pm 16.6(9) \\
69 \cdot 2 \pm 16.0(12)\end{array}$ \\
\hline
\end{tabular}

Mean \pm SD. Number of animals in parentheses. 


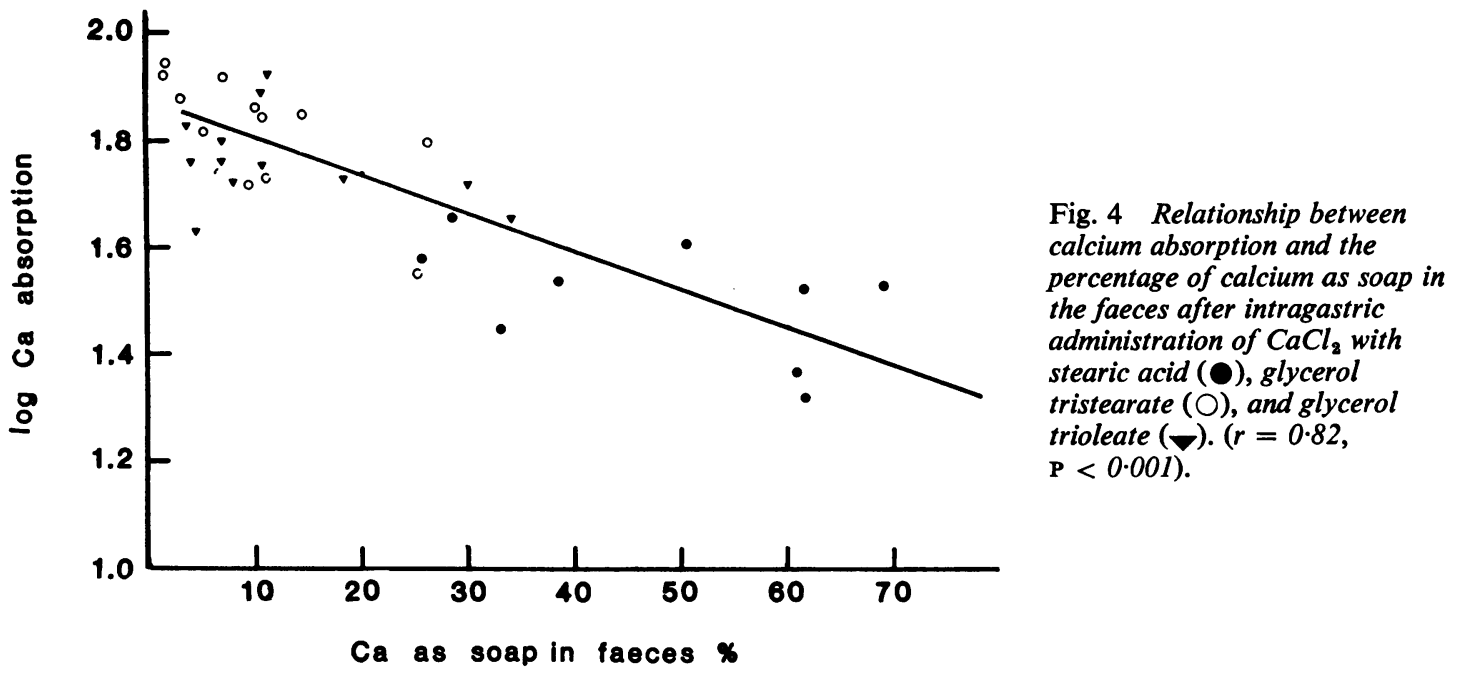

\section{Discussion}

The observation that calcium absorption from soaps is dependent on the chain length of the fatty acid is in contrast with the results of Nicolaysen (1943). This may reflect the different routes of administration of the soaps in these studies. On the basis of the results obtained it would appear that some of the fatty acids present in the normal diet are potentially able to decrease calcium absorption by soap formation. This is supported by the observed impairment of calcium absorption by stearic acid accompanied by a significant increase in Ca-soap formation in both the intestine and in the faeces. Conversely, triglycerides had little effect on calcium absorption and resulted in minimal soap formation. This may, in part, be due to incomplete hydrolysis of the triglycerides. These findings are consistent with the report that calcium absorption in rats was depressed only with extremely high triglyceride concentrations in the diet (Tadayyon and Lutwak, 1969). Similarly, in healthy man increased dietary fat content had no effect on calcium absorption (Steggerda and Mitchell, 1951).

The marked impairment of calcium absorption in the presence of phosphate is consistent with studies on milks of varying $\mathrm{Ca} / \mathrm{P}$ ratios in human infants (Barltrop and Oppé, 1973). Moreover, no interaction with simultaneously administered neutral fats was observed.

In malabsorption syndromes and in the newborn period there is often a simultaneous loss of fat and calcium in the faeces. In biliary cirrhosis the replacement of dietary fat by medium chain triglycerides has been reported to increase both fat and calcium absorption (Kehayoglou et al., 1973). Similar observations have been made in full term newborn infants (Widdowson, 1965; Williams et al., 1970). These results suggest an effect of dietary fats on calcium absorption. However, in other types of malabsorption syndromes (Agnew and Holdsworth, 1971; Harrison et al., 1973) and in premature infants (Barltrop and Oppé, 1973) the addition of well-absorbed fats to the diets were reported to increase fat absorption without having any effect on the absorption of calcium. These inconsistencies may be due to the differences in the mechanisms leading to calcium malabsorption in various conditions. Ca-soap formation may be responsible only for the reduced calcium absorption when free fatty acids or soluble soaps are present in the intestine. Thus, in pancreatic insufficiency the triglycerides are not hydrolysed and consequently no soap formation can take place, yet calcium absorption is markedly impaired (Harrison et al., 1973). Similarly, Bliss et al. (1972) found no correlation between the calcium and fat excretion in different malabsorption syndromes but a good correlation between the fatty acid and calcium soap content of the faeces.

The observation that the ratios of calcium soaps in the faeces and in the small intestine were very similar suggests that not only does their faecal content reflect the degree of soap formation in the small intestine but also suggests that no soaps are formed from the unabsorbed calcium and fatty acids in the large intestine.

G.G. was a Wellcome Research Fellow.

References

Agnew, J. E., and Holdsworth, C. D. (1971). The effect of 
fat on calcium absorption from a mixed meal in normal subjects, patients with malabsorptive disease, and patients with a partial gastrectomy. Gut, 12, 973-977.

Barltrop, D., and Oppé, T. E. (1970). Dietary factors in neonatal calcium homoeostasis. Lancet, 2, 1333-1335.

Barltrop, D., and Oppé, T. E. (1973). Absorption of fat and calcium by low birthweight infants from milks containing butterfat and olive oil. Archives of Disease in Childhood, 48, 496-501.

Bliss, C. M., Small, D. M., and Donaldson, R. M., Jr. (1972). The excretion of calcium and magnesium fatty acid soaps in steatorrhea. Gastroenterology (Abstract), 62, 724.

Boyd, O. F., Crum, C. L., and Lyman, J. F. (1932). The absorption of calcium soaps and the relation of dietary fat to calcium utilization in the white rat. Journal of Biological Chemistry, 95, 29-41.

Givens, M. H. (1917). Studies in calcium and magnesium metabolism 3. The effect of fat and fatty acid derivatives. Journal of Biological Chemistry, 31, 441-444.

Harrison, J. E., McHattie, J. D., Ligon, I. R., Jeejeebhoy, K. N., and Finlay, J. M. (1973). Effect of medium chain triglyceride on fecal calcium losses in pancreatic insufficiency. Clinical Biochemistry, 6, 136-140.

Kehayoglou, A. K., Hadziyannis, S., Kostamis, P., and Malamos, B. (1973). The effect of medium-chain triglyceride on ${ }^{47}$ calcium absorption in patients with primary biliary cirrhosis. Gut, 14, 653-656.
Kehayoglou, A. K., Holdsworth, C. D., Agnew, J. E., Whelton, M. J., and Sherlock, S. (1968). Bone disease and calcium absorption in primary biliary cirrhosis with special reference to Vitamin-D therapy. Lancet, 1, 715-719.

Kehayoglou, A. K., Williams, H. S., Whimster, W. F., and Holdsworth, C. D. (1968). Calcium absorption in the normal, bile-duct ligated, and cirrhotic rat, with observations on the effect of long- and medium-chain triglycerides. Gut, 9, 597-603.

Nicolaysen, R. (1943). The utilization of calcium soaps in rats. Acta Physiologica Scandinavica, 5, 215-218.

Sammons, H. G., and Wiggs, S. M. (1960). The separation, estimation and analysis of calcium soaps in human faeces. Clinica Chimica Acta, 5, 141-145.

Steggerda, F. R., and Mitchell, H. H. (1951). The calcium balance of adult human subjects on high- and low-fat (butter) diets. Journal of Nutrition, 45, 201-211.

Tadayyon, B., and Lutwak, L. (1969). Interrelationship of triglycerides with calcium, magnesium and phosphorus in the rat. Journal of Nutrition, 97, 246-254.

Widdowson, E. M. (1965). Absorption and excretion of fat, nitrogen, and minerals from 'filled' milks by babies one week old. Lancet, 3, 1099-1105

Williams, M. L., Rose, C. S., Morrow, G. H., Sloan, S. E., and Barness, L. A. (1970). Calcium and fat absorption in neonatal period. American Journal of Clinical Nutrition, 23, 1322-1330. 